\title{
The Use of Autogenous Dentin as a Graft in Accelerated Orthodontics Periodontally, Using the Kometa Bio Disposer
}

\section{Carrasco Gutiérrez Vicente Mtro ${ }^{1 *}$ and Macías Cid Hilda Montserrat ${ }^{2}$}

${ }^{1}$ Director of the Institute of Dental Sciences, Specialty in Oral Implantology and

Rehabilitation, Mexico

${ }^{2}$ Third Semester Resident of the Institute of Dental Sciences, Specialty in

Orthodontics, Mexico

*Corresponding Author: Carrasco Gutiérrez Vicente Mtro, Director of the Institute of Dental Sciences, Specialty in Oral Implantology and Rehabilitation, Mexico.
Received: March 15, 2021

Published: May 11, 2021

(C) All rights are reserved by Carrasco

Gutiérrez Vicente Mtro and Macías Cid

Hilda Montserrat.

\section{Abstract}

Introduction: Periodontally Accelerated Orthodontics is a procedure that helps speed up treatment. The Wilcko brothers modify the technique and, in addition to the cuts, place a graft that allows safe orthodontic movements.

Objective: To present the case of a molar Class III orthodontic patient, with a crossbite at the level of the upper left side. In addition to the use of the patient's teeth to make the bone graft.

Conclusion: The use of dental pieces as bone graft is a simple procedure, which allows a solution to many surgical situations, of course, as long as there are pieces to be extracted.

Keywords: Orthodontics; Alveolar Corticotomy; Bone Graft; Orthodontic Forces

\section{Introduction and Case Report}

Periodontally accelerated orthodontics is a clinical procedure that combines selective alveolar corticotomy, particulate bone graft and the application of orthodontic forces. Theoretically based procedure on the bone healing pattern known as regional acceleratory phenomenon (RAP), developed by the Wilcko brothers.

The periodontally accelerated orthodontic treatment combines the two major philosophies of orthodontics: natural mechanics, which refers to fixed devices, known as brackets, wires; and biological augmentation that naturally refers to the bone and gums around the tooth. The sum of both philosophies results in the treatment also known as osteogenic accelerated orthodontics.

The first works were released in 1892 by Dr. LC Bryan, however, it was until 2001 when the Wilcko brothers, Thomas, a periodon- tist and William, an orthodontist, present cases in which corticotomy was combined with graft on top of the alveoli. The objective of this work is to present an orthodontic case, resolved with the autogenous graft from human dentin, using the upper third molars of the same patient to cover the root surfaces in the upper jaw. Due to the great bone inducing potential of mineralized dental tissues, it has recently been used as a pathogen-free particulate bone graft for immediate use. The dental structure has a structure very similar to autogenous cortical bone and offers a type of graft that promotes bone formation. The dental structure has a structure very similar to autogenous cortical bone and offers a type of graft that promotes bone formation in addition to keeping the graft bed in optimal condition. In alveolar bone the inorganic content is $65 \%$ and the organic content is $25 \%$. In the end $90 \%$ of organic content is type I collagen, which plays a role outstanding in bone formation and mineralization. Protein matrix patterns in teeth have osteoconductive poten- 
tial. It is obvious why its use in the case described below. Patient taken to the consultation by a resident of the Institute of Sciences Dentists, Specialty in Orthodontics, to be attended. A 26-year-old male patient with no relevant medical history. The armed clinical examination revealed molar Class III, canine Class I, anterior edgeto-edge bite, anterior crossbite at the level of the upper left side. The study for orthodontics is requested, which includes extraoral and intraoral images, as well as orthopantomographic, lateral skull $\mathrm{x}$-rays for cephalometry and posterior anterior. It is a skeletal Class III patient, the orthopantomography shows a lesion at the apical level of the upper right central, a periapical radiograph is taken and a large lesion is seen that compromises the permanence of the tooth. Avulsion of the aforementioned tooth and corticotomy in the maxilla are performed in the same appointment. Below are the images of the entire procedure, at that time, an implant will be placed in the missing piece in a subsequent surgical event [1-5].

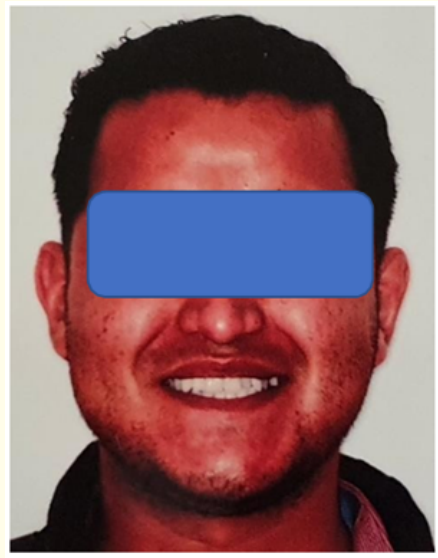

Figure 1: Front photography.

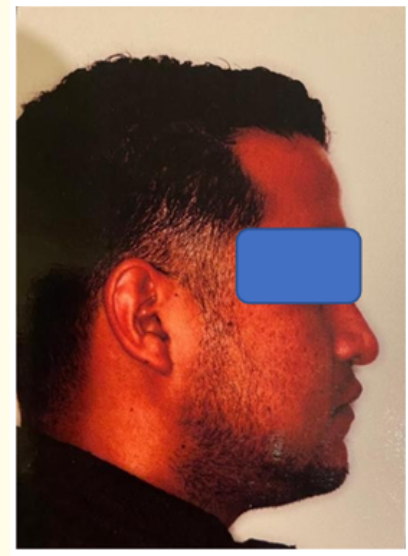

Figure 2: Right side lateral photograph.

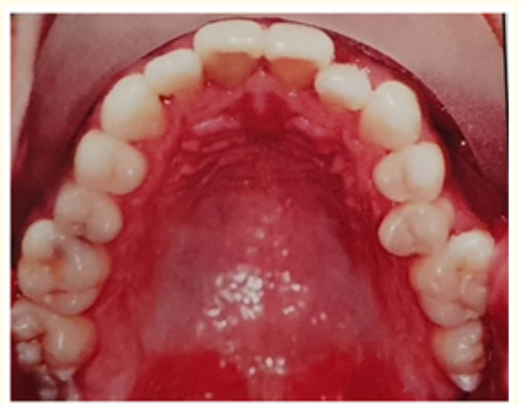

Figure 3: Intraoral photography of the maxillary arch.

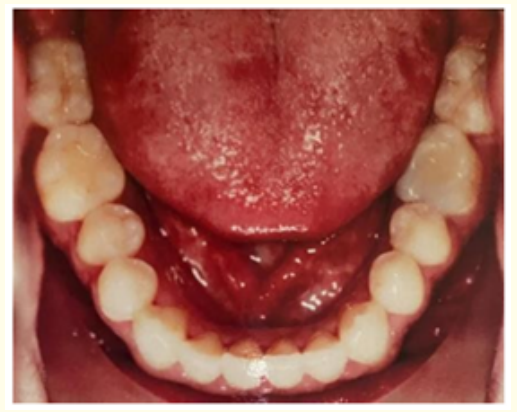

Figure 4: Intraoral photograph of the mandibular arch.
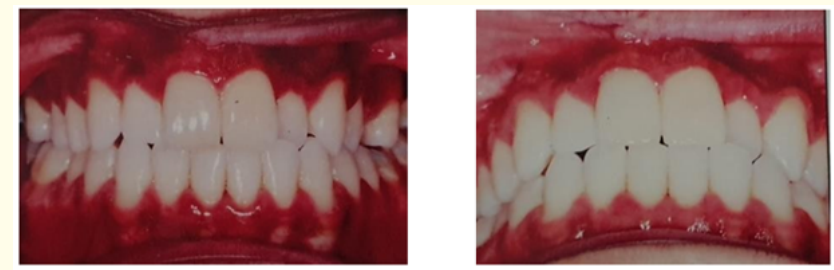

Figure 5: Intraoral bite photographs, note the situation of the upper left lateral.

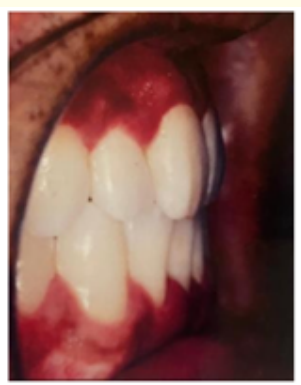

Figure 6: Overbite photography. 


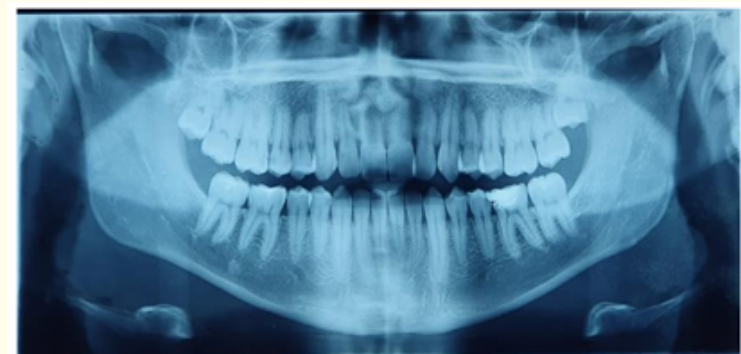

Figure 7: Orthopantomographic X-ray.

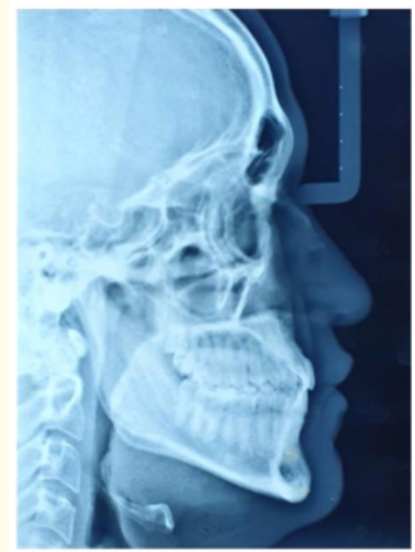

Figure 8: Lateral radiography of the skull for cephalometry.
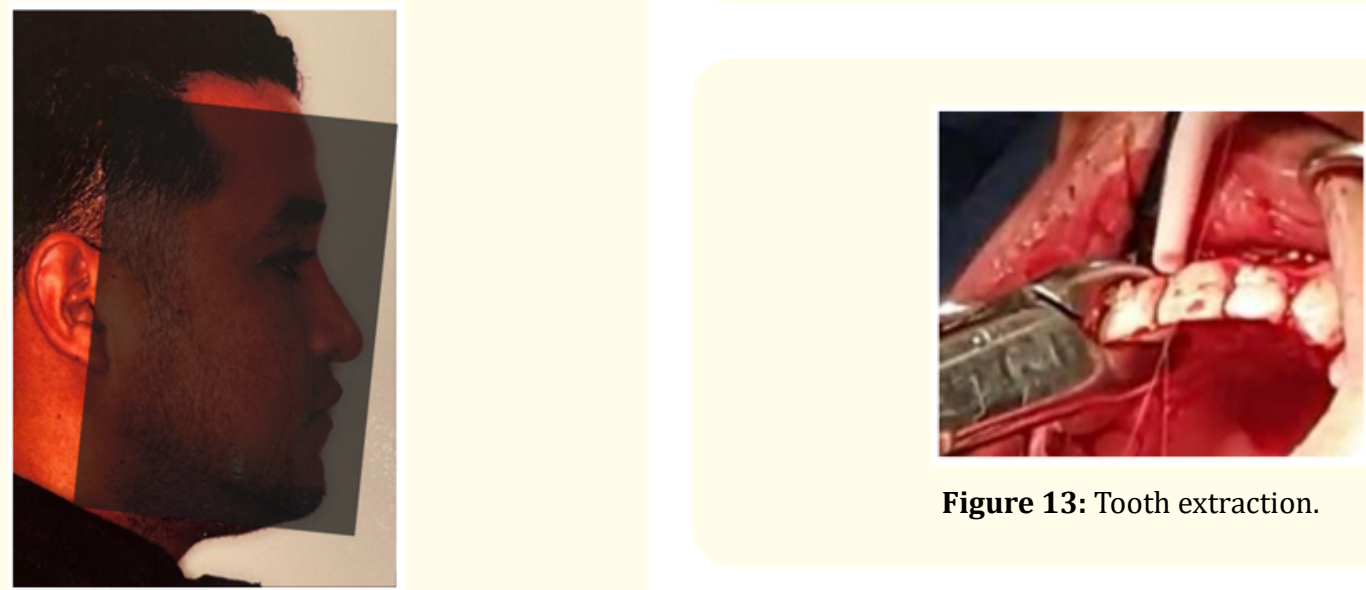

Figure 13: Tooth extraction.

Figure 9: Perez cephalometric analysis. 


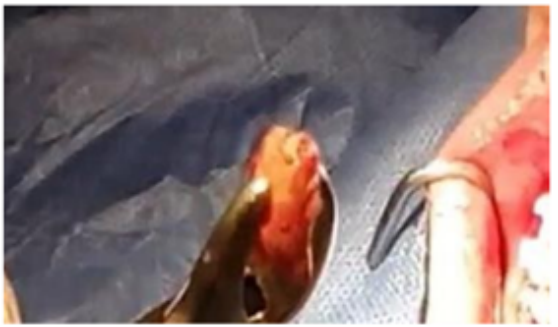

Figure 14: Note the resorption of the root tip.

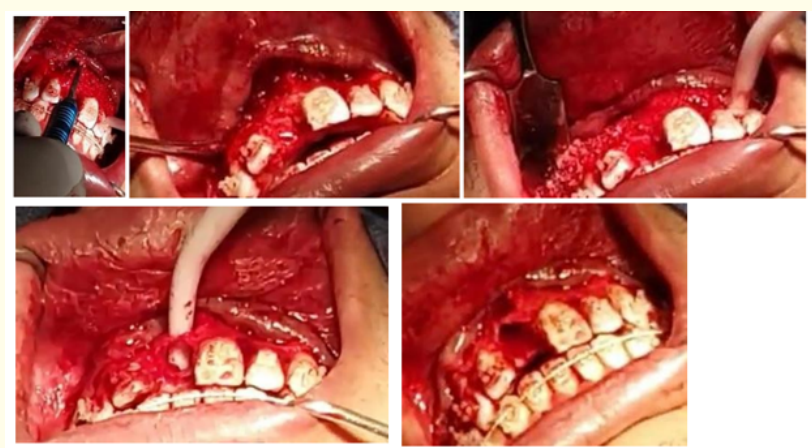

Figure 15: Note the fenestration in the external cortex at the level of the upper right center.

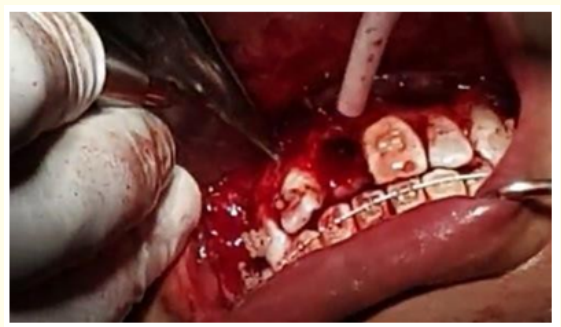

Figure 16: The use of piezoelectric for corticotomy.

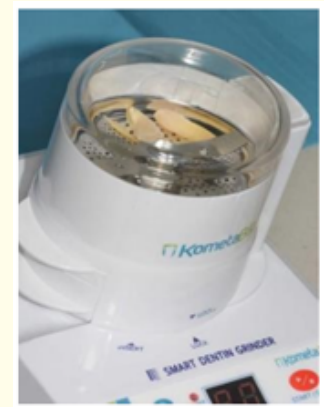

Figure 17: The Kometa Bio device.

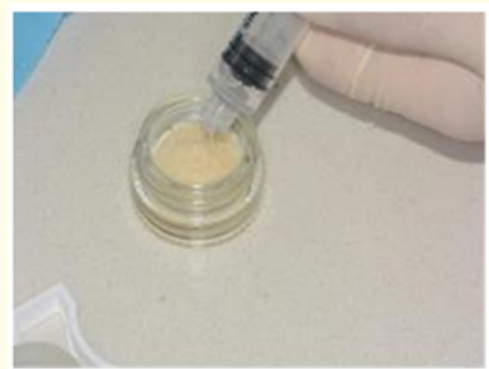

Figure 18: Preparation and conditioning of the particulate.
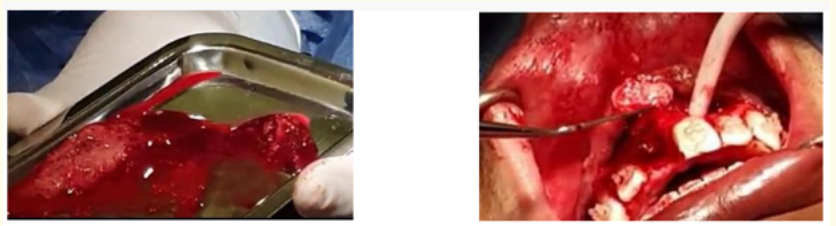

Figure 19: The autogenous graft mixed with i-PRF and brought into place.
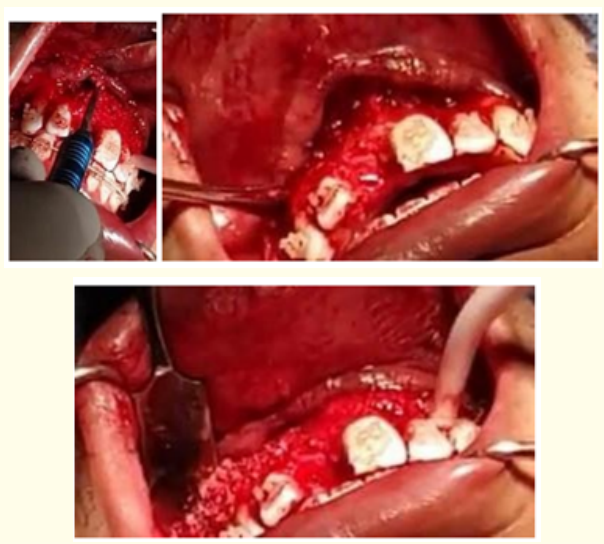

Figure 20: The graft remains in place thanks to the injectable platelet rich fibrin.

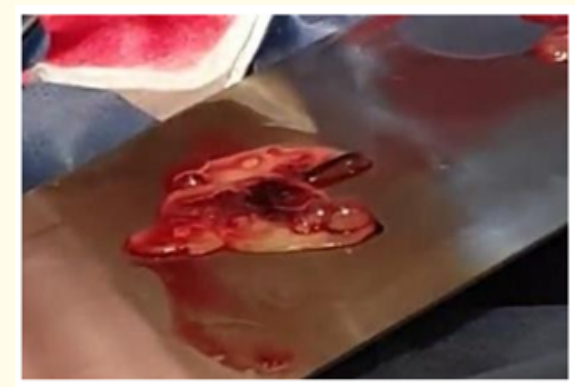




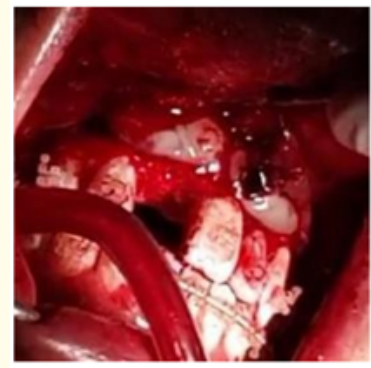

Figure 21: Placement of platelet rich fibrin membranes.

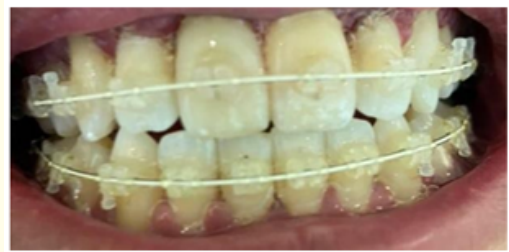

Figure 22: Result at three weeks.

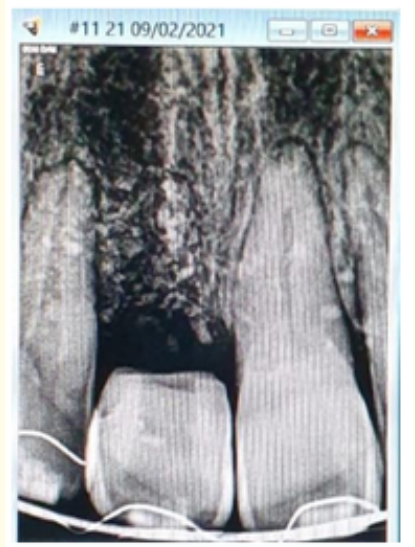

Figure 23: X-ray eight months after surgery.

\section{Bibliography}

1. Yaffe A., et al. "Regional acceleratory phenomenon in the mandible following mucoperiosteal flap surgery". Journal of Periodontology 65 (1994): 79-83.
2. Wilcko WM., et al. "Rapid orthodontics with alveolar reshaping: two case reports of decrowding". International Journal of Periodontics and Restorative Dentistry 21 (2001): 9-19.

3. Wilcko WM., et al. "Rapid orthodontic decrowding with alveolar augmentation: case report". World Journal of Orthodontics 4 (2003): 197-505.

4. Wilcko MT., et al. "The periodontally "accelerated osteogenic orthodontics "technique $\left(\mathrm{PAOO}^{\mathrm{TM}}\right)$ technique: Efficient space closing with either orthopedic or orthodontic forces". The Journal of Implant and Advanced Clinical Dentistry 1 (2009): 45-68.

5. Ferguson DJ., et al. "Selective alveolar decortication for rapid surgical-orthodontic resolution of skeletal malocclusion". Symposium on Minimally Invasive Orthognathic Surgery 63.8 (2005).

Volume 5 Issue 6 June 2021

(C) All rights are reserved by Carrasco Gutiérrez Vicente Mtro and Macías Cid Hilda Montserrat. 